\title{
Renate Damm, Rechtsanwältin
}

\author{
djb-Frauen machen Karriere - wir stellen sie vor
}

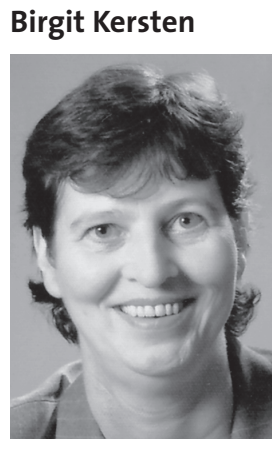

Vorstand Regionalgruppenbeirat, Vorsitzende der Regionalgruppe Oldenburg im djb, Rechtsanwältin und Vereidigte Buchprüferin, Brake
Das Interview führte Birgit Kersten, Vorstand des Regionalgruppenbeirats und Vorsitzende der RG Oldenburg im djb, Rechtsanwältin und vereidigte Buchprüferin, Brake, im November 2008 in Hamburg.

Nach dem Abitur haben Sie Jura studiert, parallel dazu haben Sie eine Theaterausbildung gemacht. Wäre auch ein ganz anderer Beruf für Sie in Frage gekommen?

Nach meinem Abitur 1955 habe ich mit dem Jurastudium angefangen. Parallel dazu habe ich in Hamburg im Studententheater mitgewirkt. Dort gab es damals einen Regisseur, Claus Peymann, der von uns verlangte, dass wir ausgebildete Schauspieler seien. Ich habe mich erst dagegen aufgelehnt, wie immer, habe dann aber die Schauspielausbildung mit zwei Schauspielern am Hamburger Schauspielhaus gemacht. Die Bühnenabschlussprüfung habe ich 1958 abgelegt. Ich habe dann während der Examensvorbereitung einen Anfängervertrag am Schauspielhaus bekommen und in den Chören von Faust II unter Gründgens gespielt.

Nachdem ich die Hausarbeit abgegeben hatte, ging das Schauspielhaus auf eine Gastspielreise nach Moskau und Petersburg, damals Leningrad, und da musste ich passen, weil genau in der Woche die Klausuren zu schreiben waren.

$\mathrm{Da}$ gab es eine wunderbare Szene zwischen Gründgens, Antje Weisgerber, der Darstellerin des Gretchens, und mir. Ich saß nämlich heulend zu Füßen von Antje Weisgerber, weil ich doch so gerne mit nach Moskau und nach Leningrad wollte. Und da kam Gründgens rein und ich heulte und heulte und da hat er gesagt: „Kind, mach das Jurastudium zu Ende, deine Rollen kommen erst ab 60“.

Und das war dann eigentlich auch das Ende meiner Schauspielerei. Ich hatte schon den großen Traum, Theater zu spielen, Schauspielerin zu werden, aber das Jurastudium stand eigentlich für mich immer im Vordergrund.

Waren Sie im Studium an einem bestimmten Bereich interessiert und haben Schwerpunkte gesetzt? Hatten Sie Vorbilder?

Ich hatte schon als Kind ein großes Vorbild, und zwar war das ein Strafverteidiger, der meinem Vater während des 2. Weltkrieges rechtlich zur Seite gestanden ist und das fand ich so faszinierend, dass ich Strafverteidigerin werden wollte. Ich habe mein Studium ganz stark auf das Strafrecht abgestellt. Ab dem zweiten Semester war ich bereits im juristischen Seminar Korrekturassisten- tin. Nach meinem 1. Staatsexamen hatte ich eine halbe Planstelle als Assistentin. Die andere halbe Planstelle hatte übrigens ein Strafrechtler, der danach einen großen Namen bekam: Claus Roxin. Er war in Hamburg für mich mein großes Vorbild.

War Axel Springer ein Mentor? Wie wichtig ist ein Mentor?

Ich glaube nicht, dass Axel Springer ein Mentor war. Ich gehe mal davon aus, dass Axel Springer

Renate Damm wurde am 27. September 1935 in Wandsbek geboren. Nach dem Abitur studierte sie Jura in Hamburg und Erlangen und machte parallel dazu eine Schauspielausbildung, die sie kurzzeitig bis auf die Bühne des Hamburger Schauspielhauses führte. 1959 legte sie ihr erstes und 1963 ihr zweites juristisches Staatsexamen ab. Danach trat sie als Syndikus-Anwältin in die Rechtsabteilung des Axel Springer Verlages ein, um ab 1967 die Leitung der Rechtsabteilung „Redaktionen“ zu übernehmen und 1985 die Leitung der Stabsabteilung Recht.

1996 wechselte Renate Damm in das Hamburger Büro der Sozietät Redeker Schön Dahs \& Sellner. Im August 2000 gründete sie gemeinsam mit Dr. Roger Mann die Anwaltssozietät Damm \& Mann. Von 1989 bis 1995 war sie Mitglied im geschäftsführenden Ausschuss der Arbeitsgemeinschaft der Syndikus-Anwälte im Deutschen Anwaltverein; bis 2001 langjähriges Mitglied im Rechtsausschuss des Verbandes Deutscher Zeitschriftenverleger e.V. (VDZ). Von 1983 bis 2002 war Renate Damm Lehrbeauftragte für Medienrecht an der Universität München und von 1992 bis 2002 Dozentin für Presseder Hochschule für Musik und Theater, Hamburg. Renate Damm ist Mitglied der Arbeitsgemeinschaft der Verlagsjustitiare sowie des Studienkreises für Presserecht und Pressefreiheit.

Renate Damm erklomm die Leiter bis ganz nach oben und wurde damit ein wichtiges „role model“. Sie hat neben dem hohen beruflichen Einsatz auch immer Zeit gefunden für das frauenpolitische Engagement: sie ist seit 1973 tätiges und bekennendes Mitglied des Deutschen Juristinnenbundes. Nach einigen Jahren im Bundesvorstand war sie von 1983 bis 1989 Erste Vorsitzende des Verbandes. recht am Institut für Kultur- und Medienmanagement 
eine solche Person weiblichen Geschlechts, wie ich es war, noch nie erlebt hatte. Es hat sich eigentlich sehr schnell herausgestellt, dass Axel Springer das auch honorierte, dass man mutig war und ich war mutig. Ich glaube, das hat ihm imponiert. Ich finde aber, dass ein Mentor sehr wichtig ist. Es gibt viel zu wenige davon. Ich habe immer Mentoring betrieben. Ein gutes Beispiel sind meine vielen Kolleginnen, die ich zum Teil ausgebildet habe und die jetzt überall in den Verlagen sind.

Axel Springer hat von Ihnen einmal gesagt, Sie seien „der einzige Mann im Haus“. Gibt es „männliche“ Eigenschaften? Wenn ja, welche und sind sie erforderlich, um Karriere zu machen?

Es ist richtig, dass Axel Springer mich einmal als den einzigen Mann im Hause Springer bezeichnet hat. Der Hintergrund war der, dass ich mich gegen die Bedenken von einigen Kollegen im Unternehmen dafür ausgesprochen hatte, eine Not-
Ehrgeiz, Strebsamkeit, sicheres Auftreten als positiv. Bei Frauen ist zum Beispiel Ehrgeiz negativ besetzt.

Erforderlich um Karriere zu machen ist für Frauen - eigentlich sehr ähnlich wie für Männer - ein Netzwerk. Ein Netzwerk, innerhalb des eigenen Unternehmens, aber auch ein Netzwerk außerhalb des Unternehmens, um sich austauschen zu können. Männer haben das automatisch. Männer haben von Anfang an, sei es im Studium, sei es nachher im beruflichen Werdegang, ihre Clubs, sie sind Mitglieder in allen möglichen beruflichen Vereinigungen. Frauen haben sich diese Position erst erkämpfen müssen. Ich weiß wovon ich rede, denn ich war in allen diesen Gremien, ob das Arbeitskreise oder auch politische Ämter waren, eigentlich fast immer überall die erste Frau. Das war leider so und das ist heute Gott sei Dank nicht mehr so.

Zum Beispiel war ich im Arbeitskreis für Presserecht und Pressefreiheit lange die einzige Frau. Dort kam ich bereits im Herbst 1962 zu der Ehre, einen Vortrag zu halten. Ich hatte das Referat zum Thema Presse und Streik für den damaligen Chefjustitiar gemacht und fühlte mich durchaus im Stande, dieses Referat selbst zu halten, weil dieser verhindert war. Das gehört eben auch zu den Dingen, die ich immer meinen jungen Kolleginnen gesagt habe: Habt den Mut zu sagen, jawohl ich kann es und tretet an. Also trat ich an und sollte zunächst nicht in den Sitzungssaal Einlass finden, weil mir gesagt wurde, da tagen jetzt die Männer. Ich habe dann doch das Referat gehalten und habe mich dann auch durchgesetzt, dass ich noch an der Diskussion teilnehmen durfte und sogar an der feierlichen Abendveranstaltung. Das war mein Start in diesem Arbeitskreis.

Wenn ich das vielleicht noch mal sagen darf: Frauen müssen, wenn sie Karriere machen wollen, wirklich in erster Linie Mut haben und die Kraft, sich auch durchzusetzen. Sie dürfen niemals klein beigeben. Das gilt natürlich eigentlich auch für Männer, nur Männer haben diesen Mut und diese Kraft von Haus aus. ausgabe von BILD Berlin zu drucken, obwohl der Verlag bestreikt wurde, allerdings mit einem Druckerei-Unternehmen, das nicht tarifgebunden war.

Natürlich gibt es männliche Eigenschaften, es gibt aber auch weibliche Eigenschaften. Ich denke, im Berufsleben unterscheiden sich die männlichen und die weiblichen Eigenschaften nicht wahnsinnig voneinander, sie werden nur anders interpretiert. Bei den männlichen Eigenschaften gelten

\section{Wie haben Sie Beruf und Familie miteinander vereinbart? Haben Sie Ihre Arbeitszeit reduziert, als Ihr Sohn geboren wurde?}

Ich habe bis zur Geburt meines Sohnes im Mai 1968 voll gearbeitet. Bis September 1968 wurde er von mir versorgt. Danach hatte ich eine Kinderpflegerin, die meinen Sohn betreute. Ich habe die Arbeitszeit im Springer Verlag nicht reduzieren wollen und auch nicht können, denn ich war damals 
schon Leiterin der Rechtsabteilung Redaktionen und hätte es mir gar nicht erlauben können, längere Zeit nur halbtags zu arbeiten. Ich wollte damals gerade einen jungen Kollegen einstellen, der fest davon ausging, dass ich nach der Schwangerschaft nicht wieder in das Unternehmen zurückkommen würde und der sich schon mein Zimmer anguckte, um es neu für sich einzurichten. Ich habe eigentlich auch nicht die Absicht gehabt, meine Arbeitszeit zu reduzieren. Jede freie Minute galt dann dem Kind, das ist ganz klar. Aber eine Teilzeitarbeit war gar nicht möglich.

Ich habe später, als ich Chefjustitiarin war, Teilzeitarbeit mit Kolleginnen, leider nie mit Kollegen, vereinbart. Ich habe das Jobsharing, wenn Sie so wollen, mit erfunden, Sekretärinnen mit Tagesmüttern versehen und auch Kolleginnen in Teilzeitarbeit miteinander arbeiten lassen, sodass sich zwei einen Arbeitsplatz richtig geteilt haben.

Sie übernahmen kurz nach Ihrer Einstellung 1967 die Leitung der Rechtsabteilung Redaktionen. 1985 wurden Sie Chefjustitiarin. Gab es für Sie überhaupt eine „Glasdecke“?

Die Leitung der Rechtsabteilung Redaktionen wurde mir deshalb angetragen, weil in der Zeit ab etwa 1966 die presserechtlichen Verfahren insbesondere gegen die BILD Zeitung unendlich zunahmen, so dass da auch eine extra Abteilung aufgebaut werden musste. Die Tatsache, dass ich erst 1985 Chefjustitiarin wurde, liegt nach meiner Einschätzung nicht daran, dass ich eine Frau war, sondern es liegt mehr daran, dass es verschiedene Rechtsabteilungen gab. Sicherlich gab es im Unternehmen Männer, die das nicht so gerne sahen, dass da eine Frau so an der Spitze stehen sollte, aber die Entwicklung war einfach durch die Unternehmenskonstruktion begründet. Erst als der Verleger Axel Springer sich entschieden hatte, die Axel Springer AG an die Börse zu bringen, wurde die Forderung laut, dass dann eine einheitliche Rechtsabteilung gebildet werden musste und es war dann der ausdrückliche Wunsch von Axel Springer, dass ich die Leitung dieser gesamten Rechtsabteilung, der Stabsabteilung Recht übernahm.

Sie wurden bei einem Bombenattentat der RAF auf das Axel Springer Haus 1972 verletzt. Wie haben Sie die Zeit erlebt?

Bei dem Bombenattentat war ich selbst ein wenig verletzt worden. Der Hintergrund hat mich damals sehr aufgewühlt, weil ich Ulrike Meinhof kannte, ich kannte auch Klaus Rainer Röhl.

Die damalige Zeit der Berichterstattung, insbesondere der BILD Zeitung, die ja auch dann letzten Endes zu den Demonstrationen einschließlich der Attentate gegen den Springer Verlag geführt haben, habe ich natürlich sehr intensiv erlebt auch innerhalb der BILD-Redaktion in Form der Diskussion mit der damaligen Chefredaktion. Das kann man heute eigentlich gar nicht mehr nachvollziehen, wie sich da die publizistische und die Seite der Demonstranten wechselseitig hochgeschaukelt haben. Ich war damals überhaupt nicht mit einigen Dingen einverstanden, habe das auch sehr deutlich gesagt, aber das konnte ich natürlich als Juristin nicht ändern, das hätten eigentlich die Redaktion ändern müssen. Heute wäre das wohl so nicht mehr möglich.

Sie haben so selbstverständlich Karriere gemacht. Was hat Sie dazu bewegt, sich im djb für andere Frauen einzusetzen und selbst junge Kolleginnen zu fördern?

Sicherlich wirkt es so nach außen, dass ich selbstverständlich Karriere gemacht habe. Ganz so war es natürlich nicht. Ich habe als Schülerin überhaupt nicht gewusst, dass Frauen und Männer ungleich behandelt werden. Im Studium habe ich dies sofort erfahren. Ich hatte mich für ein Stipendium der Studienstiftung des Deutschen Volkes beworben und musste eine Fleißprüfung machen. Der damalige Prüfer war Professor Karl Schiller. Vor ihm saßen fünf Männer und eine Frau, das war ich. Er prüfte diese fünf Männer und mich prüfte er überhaupt nicht, stellte mir keine einzige Frage. Danach gab er den fünf jungen Kollegen diese Bescheinigung. Und als ich fragte, und was ist mit mir, sagte er, sie haben ja auch nichts gesagt. Das hat dazu geführt, dass ich mich beschwert habe und er hat mich noch mal gesondert geprüft. Ich war im zweiten Semester und er hat mich im Erbrecht geprüft ... Aber ich hörte damals schon, weil ich das so interessant fand, die erbrechtlichen Vorlesungen von Prof. Leo Rape.

Schon zu Beginn meiner Berufstätigkeit im Springer Verlag habe ich erfahren müssen, dass es keinesfalls selbstverständlich war, dass man als Frau Karriere machte. Als ich nach der Geburt meines Sohnes wieder an meinen Schreibtisch zurückgekehrt war, kam als erstes die Frage eines leitenden Herren: „Müssen Sie denn noch arbeiten, verdient Ihr Mann denn so schlecht?"

Im djb habe ich feststellen müssen, dass viele Frauen nicht die gleichen Chancen für ihre Karriere hatten, die ich gehabt hatte und ich habe vor allen Dingen festgestellt, dass junge Frauen gar nicht erst versuchten, in die Wirtschaft zu gehen, weil sie sich dort sowieso keine Chancen ausrechneten. Das hat mich dazu bewegt, im djb ganz besonders Frauen zu fördern und zu unterstützen, wenn sie in die freie Wirtschaft als Justitiarinnen gingen. Ich habe in Gremien des Deutschen Anwaltvereins mitgearbeitet, da gab es eine Arbeitsgemeinschaft der Syndikusanwälte und ich habe immer versucht, Frauen in die Gremien hineinzubringen, damit diese Frauen dann auch etwas beeinflussen konnten. Ich denke, es ist eine der wichtigsten Aufgaben, wenn man selber schon einen Erfolg vorzuzeigen hat, als Vorbild für junge Kolleginnen zu dienen und ihnen auch die Angst zu nehmen, Karriere selbst zu machen und Familie und Beruf und Kinder unter einen Hut zu bekommen. In meiner Generation sind es eine ganze Menge Frauen, die das geschafft haben: Jutta Limbach, Lore Maria Peschel-Gutzeit, Heike Mundzeck oder auch Gisela Wild. Sie haben es alle geschafft und ich denke, die jungen Frauen schaffen das auch.

Wie hat sich die Berufswelt verändert, wenn Sie den Anfang Ihres Berufslebens mit heute vergleichen? Welche Ratschlä- 
ge geben Sie jungen Frauen heute, die mit einem Jurastudium Karriere machen möchten?

In der heutigen Zeit haben es junge Juristen sehr viel schwerer, sofort nach dem 2. Staatsexamen einen geeigneten Arbeitsplatz zu finden, weil die Anforderungen so hoch geworden sind, dass eigentlich nur noch Prädikatsexamina erwartet werden und möglichst soll der junge Jurist oder die junge Juristin mit 30 Jahren schon drei Fremdsprachen perfekt beherrschen und promoviert sein. Das war damals überhaupt nicht so. Wir haben eigentlich alle irgendeine berufliche Position gefunden. Ich denke, bei dieser ganzen Entwicklung ist es besonders schwer für junge Frauen, den Weg in den Beruf zu finden. Das gilt erstaunlicherweise eigentlich gar nicht so sehr für die freie Wirtschaft - in der freien Wirtschaft werden Justitiarinnen gerne eingestellt - vielmehr gilt das für die Großkanzleien. Großkanzleien stellen bevorzugt Männer ein. Genau aus dem Grunde, dass die Frauen dann ja vielleicht innerhalb der Familienplanung Kinder bekommen. Da muss man dann versuchen, sich auch in den Großkanzleien durchzusetzen. Es gibt inzwischen eine ganze Reihe von Frauen, die auch Partnerinnen in Großkanzleien sind, die sich sicherlich sehr viel mehr durchbeißen mussten, als wir dies mussten.

Ich möchte eigentlich jungen Kolleginnen raten, sich schon im Studium Gedanken darüber zu machen, was sie später beabsichtigen zu tun und sich in diese Arbeitsbereiche hineinzufinden, auch Praktika in Anwaltskanzleien machen. Ich bleibe jetzt nur beim Presserecht: An den großen Gerichten gibt es spezielle Pressekammern und spezielle Pressesenate bei den jeweiligen Oberlandesgerichten. Um dorthin zu kommen, muss man mit Beginn der Referendarzeit die Stationen planen und von vornherein sagen, ich möchte dorthin.

Sie waren von 1983 bis 1989 Vorsitzende des djb und haben in verschiedenen Kommissionen (Familienrecht, Strafrecht, Steuerrecht, Gentechnologie) mitgearbeitet. Welche Reformen und Gesetze, die Sie begleitet haben, waren Ihnen besonders wichtig? Welche Frauen haben Sie besonders beeinflusst und angeregt bei Ihrer Arbeit? Haben Sie einen „Nutzen“ aus Ihrer Tätigkeit im djb gezogen?

In meiner Tätigkeit habe ich ganz besonders von dem Wissen der anderen Kolleginnen profitiert. Ich nenne da wieder Lore Maria Peschel-Gutzeit und Jutta Limbach. Ganz besonders habe ich mich um die juristischen Probleme der Gentechnologie gekümmert. Das war einfach ein Rechtsgebiet, wo der djb aufgerufen war, etwas mit zu gestalten und das haben wir dann ja auch getan. Ein sehr großes persönliches Anliegen war für mich die Änderung des ehelichen Namenrechts. Ich war immer eine Gegnerin dieser entsetzlichen Doppelnamen.

Abgesehen von der Tatsache, dass ich im djb eine ganze Fülle von neuen Ideen bekommen habe und eine Anzahl von Kolleginnen kennen gelernt habe, die ich sonst nie kennen gelernt hätte, kann ich wirklich für mich sagen, dass der Nutzen aus der Arbeit im djb für mich in der täglichen Arbeit auch heute immer noch vorhanden ist. Wie im djb miteinander umgegangen wurde, war für mich sehr prägend.

Thema im djb ist wieder einmal die Quote. Bereits vor 20 Jahren, anlässlich des 40-jährigen Jubiläums, wurde eine "Quote $X$ “ gefordert, d.h. ein der Organisation oder dem Lebensbereich entsprechender Frauenanteil. Sie waren damals Vorsitzende des djb und haben die Quote befürwortet.

Ich bin nach wie vor eine glühende Anhängerin der Frauenquote. Ich habe festgestellt, dass ohne die Quote in der Vergangenheit nicht allzu viel bewegt worden wäre. Die Quote hat doch einiges bewirkt,

\section{Erforderlich ist für Frauen, um Karriere zu machen, ein Netzwerk. Männer haben von Anfang an ihre Clubs, sie sind Mitglieder in allen möglichen Vereinigungen. Frau- en haben sich diese Position erst erkämpfen müssen.}

auch wenn sich damals, als ich die Quote vertreten habe, viele über mich aufgeregt oder mich belächelt haben. Ich bin der Meinung, die Quote ist als Druckmittel erforderlich, um überhaupt etwas zu bewirken. Ich würde es gerne noch erleben, dass die Quote nicht mehr erforderlich wäre. Aber ich fürchte, das wird so schnell nicht der Fall sein. Gerade in den Vorständen der Wirtschaftsunternehmen und vor allen Dingen in den Aufsichtsräten gibt es viel zu wenige Frauen. Und wenn Frauen wirklich einmal in einem Vorstand angekommen sind, dann werden sie auch sehr schnell wieder rausgemobbt oder arbeiten so viel, dass sie keine Zeit mehr haben, überhaupt noch zu leben. Und die Aufsichtsräte, also, das ist für mich noch wirklich eine wahre Männerwüste.

In meiner Zeit als Chefjustitiarin hatte ich es tatsächlich erreicht, dass ich einen Frauenanteil bei den Justitiarinnen von 50 Prozent hatte, was den Männern nicht unbedingt gefiel. Dieses habe ich auch immer sehr nach außen vertreten, weil ich der Meinung war, dass Unternehmen sich damit auch 
schmücken konnten, viele Frauen zu beschäftigen. Ich habe Frauen in Unternehmen auch wirklich immer in die eigene Verantwortung entlassen, sie alleine in die Sitzungen gehen lassen, auch wenn sie ganz jung bei mir angefangen hatten. Ich habe immer versucht, sie aufzubauen: „Du kannst das, du gehst jetzt in die Sitzung, du machst das schon.“

Sie waren Justitiarin, sind Lehrbeauftragte, Dozentin, Autorin, Politikerin und Rechtsanwältin. Sie haben das Bundesverdienstkreuz 1. Klasse für Ihr Engagement im djb erhalten. Gibt es neue Bereiche, die Sie reizen?

Mich reizen eigentlich immer wieder neue rechtliche Bereiche. Ein Bereich, den ich gerade jetzt entdeckt habe, ist eine Frage aus Art. 5 GG, nämlich die Kunstfreiheit. Ich möchte gerne untersuchen, ob die Kunstfreiheit tatsächlich schrankenlos sein soll oder ob nicht die Schranken des Art. 5 Abs. 2 GG zumindest indirekt auch für die Kunstfreiheit gelten müssen. Ich kann nicht einsehen, dass die Kunstfreiheit, wie im Fall Esra, dazu führen kann, dass die Intimsphäre einer Frau und auch die Privatsphäre der Mutter ganz schwer verletzt werden. Das Bundesverfassungsgericht hat in der Entscheidung die Verletzung des allgemeinen Persönlichkeitsrechtes der Mutter nicht so schwerwiegend angesehen und hat insoweit ihrer Klage nicht stattgegeben und nur Esra den Anspruch zugebilligt, den Roman verbieten zu lassen. Wenn eine Zeitung etwas Derartiges über die Mutter schreiben würde, würde das sofort verboten werden.

Ich persönlich habe jetzt noch etwas vor: ich werde meine seit 1990 auf Eis liegende Promotion zum Thema der recht- lichen Probleme der Zeitungen und Zeitschriften einschließlich des Pressevertriebes im Zusammenhang mit der Wende schreiben. Ich habe damals selber in vielen dieser Verhandlungen um den Pressevertrieb Ost und auch um die Pläne der westdeutschen Verlage, die ostdeutsche Zeitungsverlage zu übernehmen, mitgearbeitet und ich habe vor allen Dingen einen der wichtigsten Prozesse in diesem Bereich selbst geführt. Es war der Titelschutzprozess BZ, gleich Berliner Zeitung West, gegen Berliner Zeitung Ost. Ich möchte das alles gerne noch einmal aufarbeiten im Rahmen dieser Dissertation.

\section{Wie bekommen Sie Abstand von Ihrem Beruf? Brauchen Sie Abstand? Setzt sich Ihre Dynamik auch außerhalb Ihres Beru- fes fort?}

Ich habe es immer verstanden, Abstand vom Beruf zu haben. Dabei hat mir natürlich auch meine Theaterleidenschaft immer geholfen. Ich kann aber auch ganz anders abschalten. Ich habe seit über 33 Jahren eine Skihütte in Österreich und ich bin nach wie vor leidenschaftliche Abfahrtsfahrerin. Ich bin sehr begeistert von unserem Leben auf Mallorca. Ich kann einfach einen Schalter umlegen. Ob meine Dynamik sich auch außerhalb des Berufes fortsetzt, das glaube ich eigentlich nicht. Ich brauche auch für mich mal ruhige Phasen und die finde ich vor allen Dingen auf Mallorca in unserem Haus und ganz besonders in meinem eigenen Maleratelier. Seit meiner Kinderzeit male ich. Und wenn ich male, ist die Welt um mich total versunken.

Frau Damm, ich danke Ihnen für das Gespräch.

\section{Impressum}

\author{
Schriftleitung: \\ Anke Gimbal, Rechtsassessorin (V.i.S.d.P.) \\ Juliane Lindner \\ Redaktionsanschrift: \\ Deutscher Juristinnenbund e. V. \\ Anklamer Str. 38 \\ 10115 Berlin \\ Telefon: 030 443270-0 \\ Telefax: 030 443270-22 \\ E-Mail: geschaeftsstelle@djb.de
}

\section{Druck und Verlag:}

Nomos Verlagsgesellschaft mbH \& Co. KG

Waldseestr. 3-5

D-76530 Baden-Baden

Telefon: 07221 2104-0

Telefax: 07221 2104-27

\author{
Anzeigenverwaltung und \\ Anzeigenannahme: \\ Sales friendly, Verlagsdienstleistungen \\ Bettina Roos \\ Siegburgerstr. 123 \\ D-53229 Bonn \\ Telefon: 0228 97898-o \\ Telefax: 0228 97898-20 \\ E-Mail: roos@sales-friendly.de
}

Die Zeitschrift sowie alle in ihr enthaltenen einzelnen Beiträge und Abbildungen sind urheberrechtlich geschützt. Jede Verwertung, die nicht ausdrücklich vom Urheberrechtsgesetz zugelassen ist, bedarf der vorherigen Zustimmung des Verlages.

Namentlich gekennzeichnete Artikel müssen nicht die Meinung des Herausgebers oder der Schriftleitung wiedergeben. Unverlangt eingesendete Manuskripte - für die keine Haftung übernommen wird - gelten als Veröffentli- chungsvorschlag zu den Bedingungen des Verlags. Es werden nur unveröffentlichte Originalarbeiten angenommen. Die Verfasser erklären sich mit einer nicht sinnentstellenden redaktionellen Bearbeitung einverstanden.

\author{
Erscheinungsweise: \\ vierteljährlich \\ Bezugspreis 2008: \\ jährlich 48,-€, Einzelheft 13,-€
}

Alle Preise zzgl. Vertriebs-Direktbeordnungsgebühren inkl. MWSt.;

Bestellungen nehmen entgegen:

Der Buchhandel und der Verlag; Kündigung jeweils drei Monate zum Kalenderjahresende. Zahlungen jeweils im Voraus an: Nomos Verlagsgesellschaft, Postbank Karlsruhe, Konto 7363651 (BLZ 66010075) oder Stadtsparkasse Baden-Baden, Konto 5002266 (BLZ 66250030). 